Supplement of Atmos. Chem. Phys., 14, 9137-9153, 2014

http://www.atmos-chem-phys.net/14/9137/2014/

doi:10.5194/acp-14-9137-2014-supplement

(C) Author(s) 2014. CC Attribution 3.0 License.

(c) (1)

Supplement of

\section{Ozone vegetation damage effects on gross primary productivity in the} United States

X. Yue and N. Unger

Correspondence to: X. Yue (xu.yue@yale.edu)

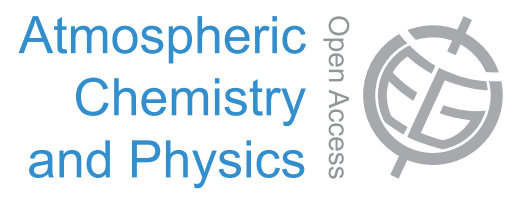


Table S1. Match of model PFTs with that from NACP sites and the ISLSCP dataset.

\begin{tabular}{|c|c|c|c|c|c|}
\hline \multicolumn{2}{|c|}{ PFTs in the model } & \multicolumn{2}{|c|}{ PFTs at NACP sites } & \multicolumn{2}{|c|}{ PFTs from ISLSCP } \\
\hline ID & PFTs & ID & PFTs & ID & PFTs \\
\hline 1 & Tundra & & $\mathrm{N} / \mathrm{A}$ & & $\mathrm{N} / \mathrm{A}$ \\
\hline 2 & $\mathrm{C} 3$ grassland & I & Grasslands & $\mathrm{a}$ & Grasslands \\
\hline 3 & $\mathrm{C} 4$ grassland & & N/A & $\mathrm{b}$ & Savannas \\
\hline \multirow[t]{4}{*}{4} & Shrubland & II & Closed Shrubland & $\mathrm{c}$ & Closed Shrubland \\
\hline & & III & Woody Savannas & $\mathrm{d}$ & Open Shrubland \\
\hline & & IV & Permanent Wetlands & $\mathrm{e}$ & Woody Savannas \\
\hline & & & & $\mathrm{f}$ & Permanent Wetlands \\
\hline \multirow[t]{2}{*}{5} & Deciduous Broadleaf Forest & $\mathrm{V}$ & Deciduous Broadleaf Forest & $\mathrm{g}$ & Deciduous Broadleaf Forest \\
\hline & & & & $\mathrm{h}$ & Deciduous Needleleaf Forest \\
\hline \multirow[t]{2}{*}{6} & Evergreen Needleleaf Forest & VI & Evergreen Needleleaf Forest & $\mathrm{i}$ & Evergreen Needleleaf Forest \\
\hline & & VII & Mixed Forests & $\mathrm{j}$ & Mixed Forests \\
\hline 7 & Tropical Rainforest & & $\mathrm{N} / \mathrm{A}$ & $\mathrm{k}$ & Evergreen Broadleaf Forest \\
\hline \multirow[t]{2}{*}{8} & Cropland & VIII & Croplands (C4) & 1 & Croplands (C3/C4) \\
\hline & & & & $\mathrm{m}$ & Cropland/Natural vegetation mosaic $(\mathrm{C} 3 / \mathrm{C} 4)$ \\
\hline
\end{tabular}




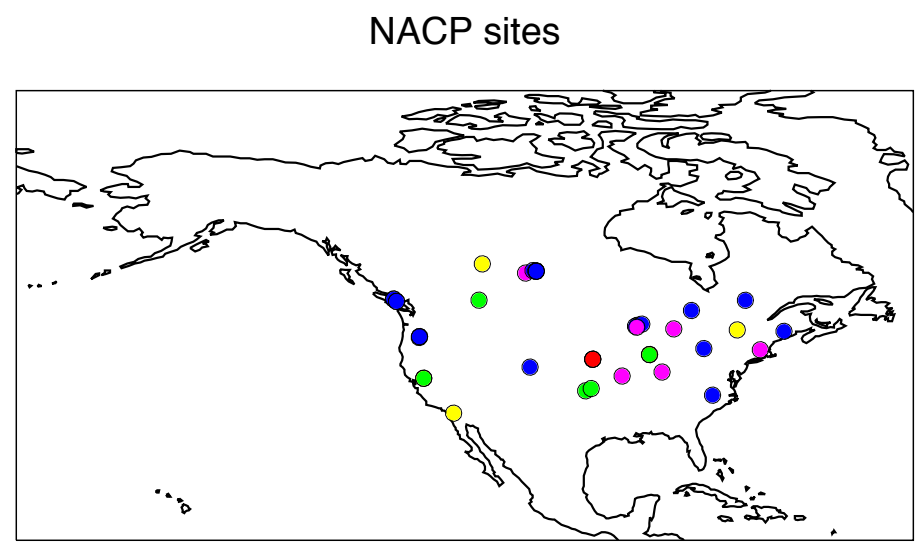

Fig. S1. Distribution of 40 North American Carbon Program (NACP) sites. The color indicates different land types as evergreen needleleaf forest (ENF, blue), deciduous broadleaf forest (DBF, magenta), grasslands (GRA, green), croplands (CRO, red), shrublands (SHR, yellow). "Mixed Forests" are classified as ENF, "Permanent Wetlands" and "Woody Savannas" as SHR (Table S1). The local vegetation type at each site is described in appendix Table A. 


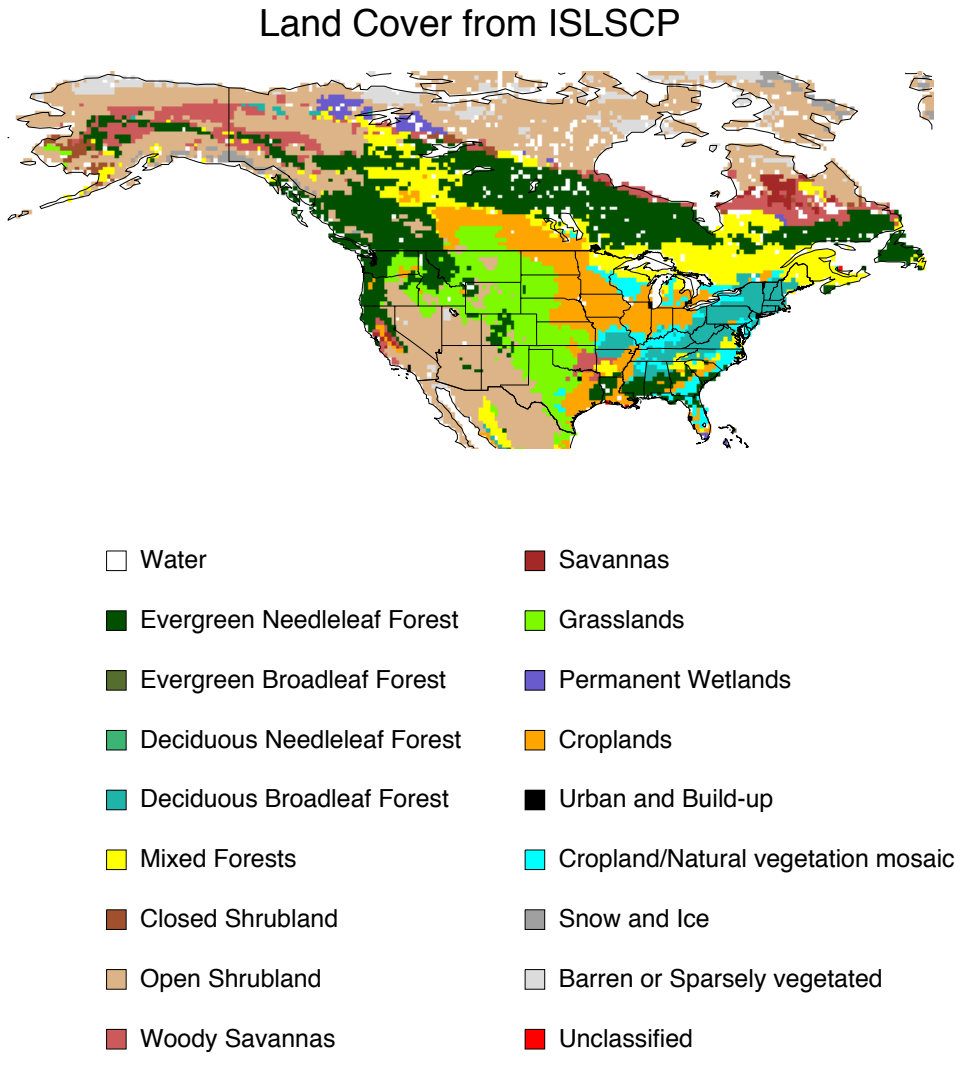

Fig. S2. Land cover types in North America developed by the International Satellite Land-Surface Climatology Project (ISLSCP). 


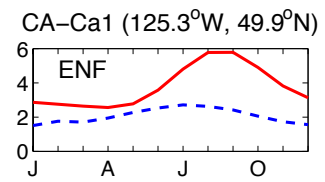

CA-NS1 $\left(124.9^{\circ} \mathrm{W}, 49.5^{\circ} \mathrm{N}\right)$

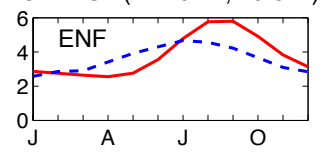

CA-SJ1 $\left(104.7^{\circ} \mathrm{W}, 53.9^{\circ} \mathrm{N}\right)$

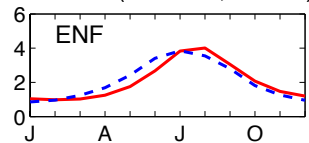

US-Dk3 $\left(79.1^{\circ} \mathrm{W}, 36^{\circ} \mathrm{N}\right)$

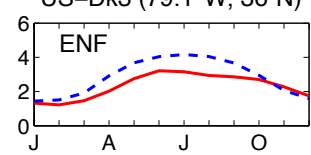

US-Me5 $\left(121.6^{\circ} \mathrm{W}, 44.4^{\circ} \mathrm{N}\right)$

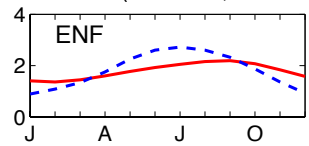

CA-Oas $\left(106.2^{\circ} \mathrm{W}, 53.6^{\circ} \mathrm{N}\right)$

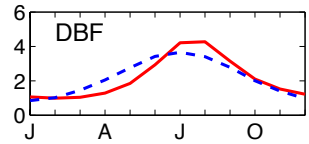

US-UMB $\left(84.7^{\circ} \mathrm{W}, 45.6^{\circ} \mathrm{N}\right)$

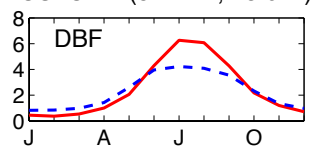

US-Los $\left(90^{\circ} \mathrm{W}, 46.1^{\circ} \mathrm{N}\right)$

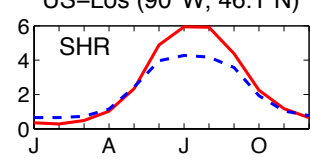

US-ARM $\left(97.5^{\circ} \mathrm{W}, 36.6^{\circ} \mathrm{N}\right)$

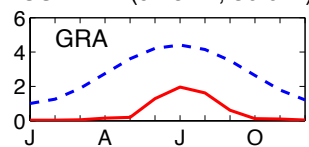

US-IB1 $\left(88.2^{\circ} \mathrm{W}, 41.9^{\circ} \mathrm{N}\right)$

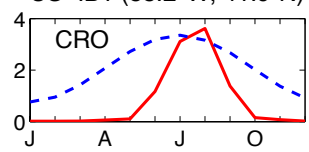

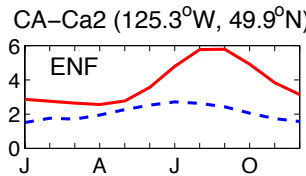

CA-Obs $\left(105.1^{\circ} \mathrm{W}, 54^{\circ} \mathrm{N}\right)$

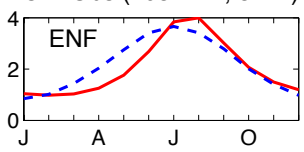

CA-SJ2 $\left(104.6^{\circ} \mathrm{W}, 53.9^{\circ} \mathrm{N}\right)$

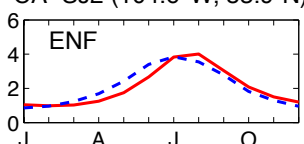

US-Ho1 $\left(68.7^{\circ} \mathrm{W}, 45.2^{\circ} \mathrm{N}\right)$

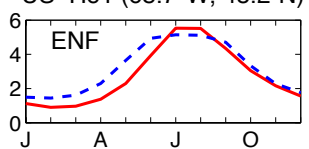

US-NR1 $\left(105.5^{\circ} \mathrm{W}, 40^{\circ} \mathrm{N}\right)$

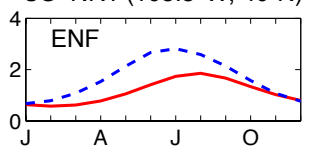

US-Ha1 $\left(72.2^{\circ} \mathrm{W}, 42.5^{\circ} \mathrm{N}\right)$

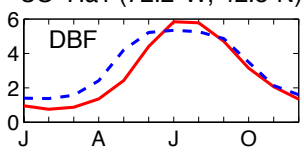

US-WCr $\left(90.1^{\circ} \mathrm{W}, 45.8^{\circ} \mathrm{N}\right)$

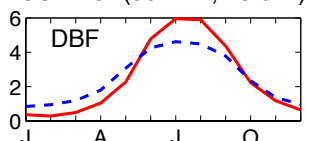

US-SO2 $\left(116.6^{\circ} \mathrm{W}, 33.4^{\circ} \mathrm{N}\right)$

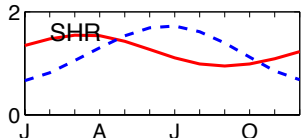

US-IB2 $\left(88.2^{\circ} \mathrm{W}, 41.8^{\circ} \mathrm{N}\right)$

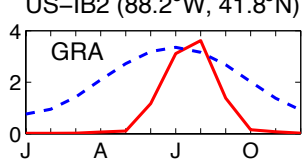

US-Ne1 $\left(96.5^{\circ} \mathrm{W}, 41.2^{\circ} \mathrm{N}\right)$

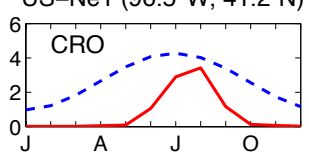

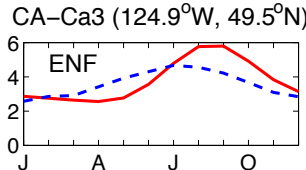

CA-Ojp $\left(104.7^{\circ} \mathrm{W}, 53.9^{\circ} \mathrm{N}\right)$

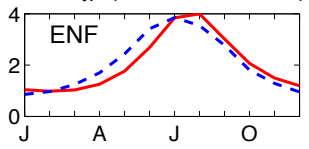

CA-SJ3 $\left(104.6^{\circ} \mathrm{W}, 53.9^{\circ} \mathrm{N}\right)$

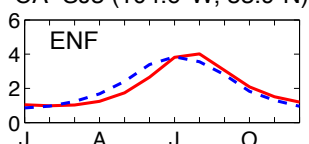

US-Me2 $\left(121.6^{\circ} \mathrm{W}, 44.5^{\circ} \mathrm{N}\right)$
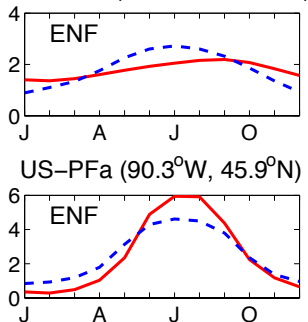

US-MMS $\left(86.4^{\circ} \mathrm{W}, 39.3^{\circ} \mathrm{N}\right)$

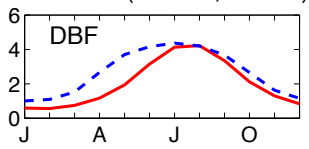

CA-Mer $\left(75.5^{\circ} \mathrm{W}, 45.4^{\circ} \mathrm{N}\right)$

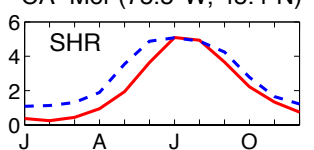

US-Ton $\left(121^{\circ} \mathrm{W}, 38.4^{\circ} \mathrm{N}\right)$

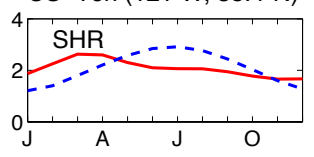

US-Shd $\left(96.7^{\circ} \mathrm{W}, 36.9^{\circ} \mathrm{N}\right)$

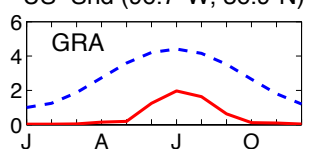

US-Ne2 $\left(96.5^{\circ} \mathrm{W}, 41.2^{\circ} \mathrm{N}\right)$

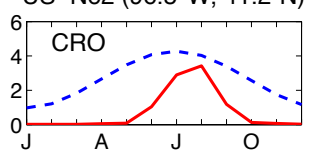

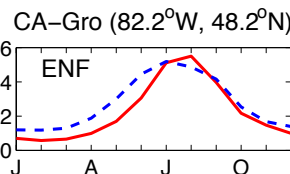

CA-Qfo $\left(74.3^{\circ} \mathrm{W}, 49.7^{\circ} \mathrm{N}\right)$

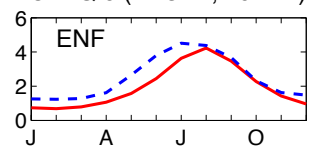

CA-TP4 $\left(80.4^{\circ} \mathrm{W}, 42.7^{\circ} \mathrm{N}\right)$

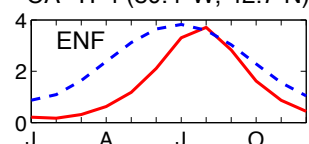

US-Me3 $\left(121.6^{\circ} \mathrm{W}, 44.3^{\circ} \mathrm{N}\right)$

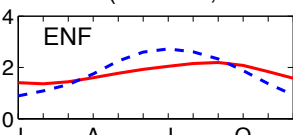

US-Syv $\left(89.3^{\circ} \mathrm{W}, 46.2^{\circ} \mathrm{N}\right)$

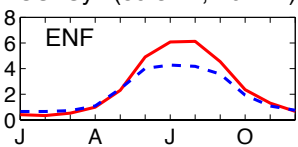

US-MOz $\left(92.2^{\circ} \mathrm{W}, 38.7^{\circ} \mathrm{N}\right)$

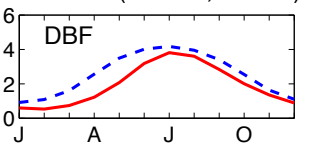

CA-WP1 $\left(112.5^{\circ} \mathrm{W}, 55^{\circ} \mathrm{N}\right)$

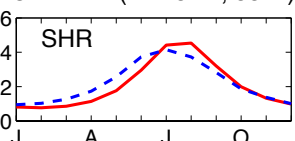

CA-Let $\left(112.9^{\circ} \mathrm{W}, 49.7^{\circ} \mathrm{N}\right)$

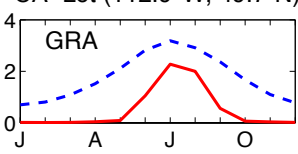

US-Var $\left(121^{\circ} \mathrm{W}, 38.4^{\circ} \mathrm{N}\right)$

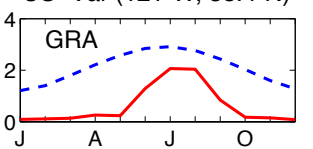

US-Ne3 $\left(96.4^{\circ} \mathrm{W}, 41.2^{\circ} \mathrm{N}\right)$

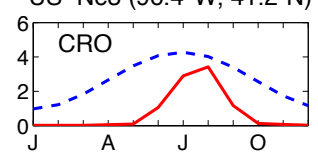

Fig. S3. Comparison of monthly mean leaf area index (LAI, $\mathrm{m}^{2} \mathrm{~m}^{-2}$ ) from the Modern Era-Retrospective Analysis (MERRA) reanalysis (red solid lines) and the Moderate Resolution Imaging Spectroradiometer (MODIS) (blue dashed lines) at each NACP site. The name and location (longitude, latitude) are shown in the title. The land types include evergreen needleleaf forest (ENF), deciduous broadleaf forest (DBF), shrublands (SHR), grasslands (GRA), and croplands (CRO). 


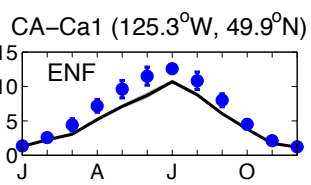

CA-NS1 $\left(124.9^{\circ} \mathrm{W}, 49.5^{\circ} \mathrm{N}\right)$

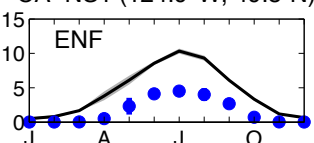

CA-SJ1 $\left(104.7^{\circ} \mathrm{W}, 53.9^{\circ} \mathrm{N}\right)$

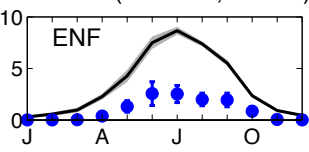

US-Dk3 $\left(79.1^{\circ} \mathrm{W}, 36^{\circ} \mathrm{N}\right)$

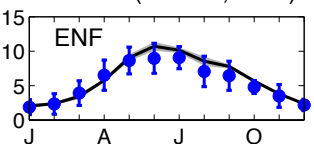

US-Me5 $\left(121.6^{\circ} \mathrm{W}, 44.4^{\circ} \mathrm{N}\right)$

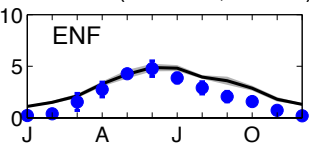

CA-Oas $\left(106.2^{\circ} \mathrm{W}, 53.6^{\circ} \mathrm{N}\right)$

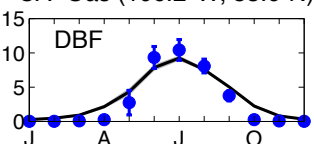

US-UMB $\left(84.7^{\circ} \mathrm{W}, 45.6^{\circ} \mathrm{N}\right)$

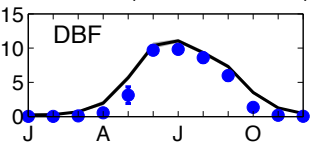

US-Los $\left(90^{\circ} \mathrm{W}, 46.1^{\circ} \mathrm{N}\right)$

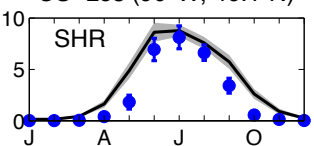

US-ARM $\left(97.5^{\circ} \mathrm{W}, 36.6^{\circ} \mathrm{N}\right)$

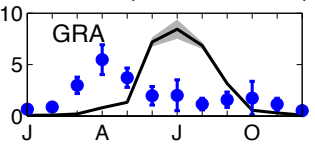

US-IB1 $\left(88.2^{\circ} \mathrm{W}, 41.9^{\circ} \mathrm{N}\right)$

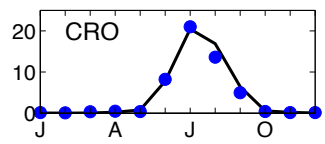

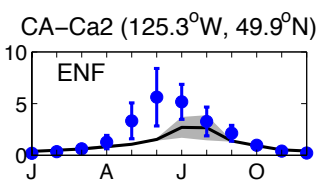

CA-Obs $\left(105.1^{\circ} \mathrm{W}, 54^{\circ} \mathrm{N}\right)$

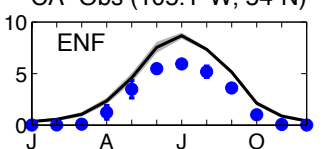

CA-SJ2 $\left(104.6^{\circ} \mathrm{W}, 53.9^{\circ} \mathrm{N}\right)$

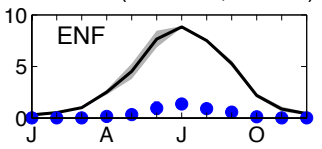

US-Ho1 $\left(68.7^{\circ} \mathrm{W}, 45.2^{\circ} \mathrm{N}\right)$
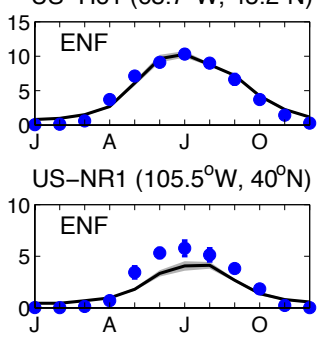

US-Ha1 $\left(72.2^{\circ} \mathrm{W}, 42.5^{\circ} \mathrm{N}\right)$

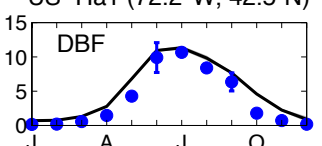

US-WCr $\left(90.1^{\circ} \mathrm{W}, 45.8^{\circ} \mathrm{N}\right)$

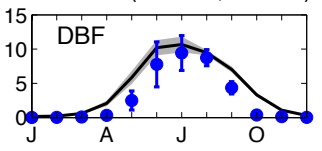

US-SO2 $\left(116.6^{\circ} \mathrm{W}, 33.4^{\circ} \mathrm{N}\right)$

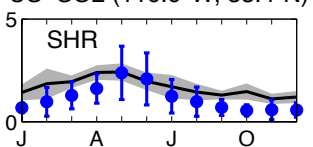

US-IB2 $\left(88.2^{\circ} \mathrm{W}, 41.8^{\circ} \mathrm{N}\right)$

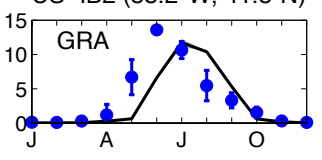

US-Ne1 $\left(96.5^{\circ} \mathrm{W}, 41.2^{\circ} \mathrm{N}\right)$

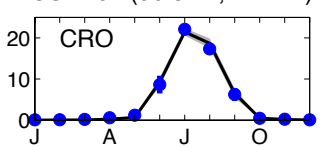

$\mathrm{CA}-\mathrm{Ca} 3\left(124.9^{\circ} \mathrm{W}, 49.5^{\circ} \mathrm{N}\right)$

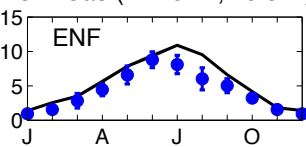

CA-Ojp $\left(104.7^{\circ} \mathrm{W}, 53.9^{\circ} \mathrm{N}\right)$

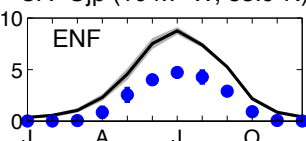

CA-SJ3 $\left(104.6^{\circ} \mathrm{W}, 53.9^{\circ} \mathrm{N}\right)$

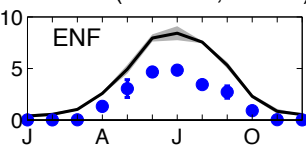

US-Me2 $\left(121.6^{\circ} \mathrm{W}, 44.5^{\circ} \mathrm{N}\right)$

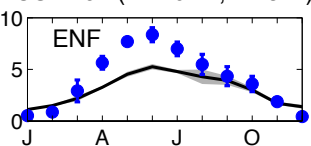

US-PFa $\left(90.3^{\circ} \mathrm{W}, 45.9^{\circ} \mathrm{N}\right)$

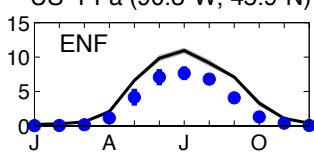

US-MMS $\left(86.4^{\circ} \mathrm{W}, 39.3^{\circ} \mathrm{N}\right)$

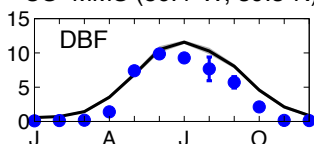

CA-Mer $\left(75.5^{\circ} \mathrm{W}, 45.4^{\circ} \mathrm{N}\right)$

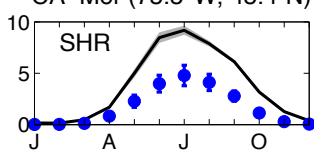

US-Ton $\left(121^{\circ} \mathrm{W}, 38.4^{\circ} \mathrm{N}\right)$

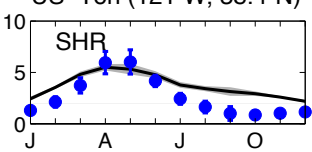

US-Shd $\left(96.7^{\circ} \mathrm{W}, 36.9^{\circ} \mathrm{N}\right)$

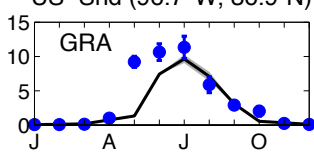

US-Ne2 $\left(96.5^{\circ} \mathrm{W}, 41.2^{\circ} \mathrm{N}\right)$

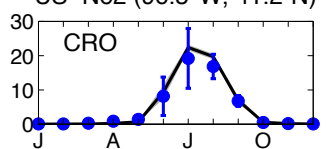

CA-Gro $\left(82.2^{\circ} \mathrm{W}, 48.2^{\circ} \mathrm{N}\right)$

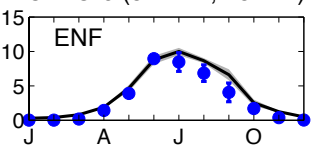

CA-Qfo $\left(74.3^{\circ} \mathrm{W}, 49.7^{\circ} \mathrm{N}\right)$

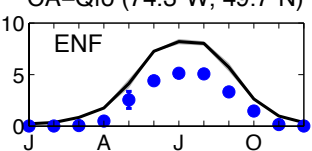

CA-TP4 $\left(80.4^{\circ} \mathrm{W}, 42.7^{\circ} \mathrm{N}\right)$

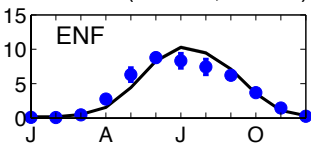

US-Me3 $\left(121.6^{\circ} \mathrm{W}, 44.3^{\circ} \mathrm{N}\right)$

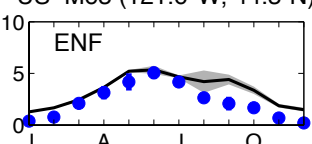

US-Syv $\left(89.3^{\circ} \mathrm{W}, 46.2^{\circ} \mathrm{N}\right)$

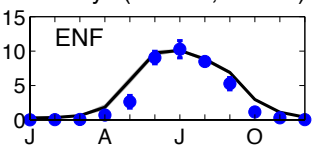

US-MOz $\left(92.2^{\circ} \mathrm{W}, 38.7^{\circ} \mathrm{N}\right)$

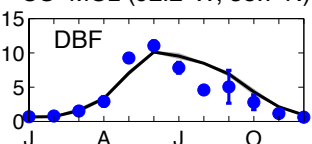

CA-WP1 $\left(112.5^{\circ} \mathrm{W}, 55^{\circ} \mathrm{N}\right)$

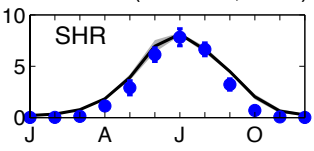

CA-Let $\left(112.9^{\circ} \mathrm{W}, 49.7^{\circ} \mathrm{N}\right)$

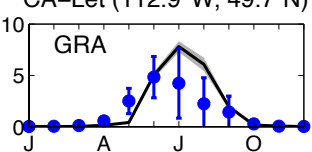

US-Var $\left(121^{\circ} \mathrm{W}, 38.4^{\circ} \mathrm{N}\right)$

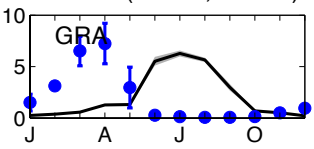

US-Ne3 $\left(96.4^{\circ} \mathrm{W}, 41.2^{\circ} \mathrm{N}\right)$

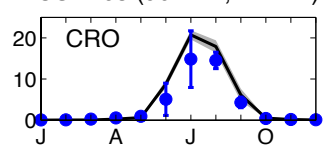

Fig. S4. Comparison of long-term monthly mean GPP $\left(\mathrm{g}[\mathrm{C}] \mathrm{m}^{-2} \mathrm{day}^{-1}\right)$ from observations (blue points with error bars indicating one standard deviation) and the $\mathrm{O}_{3^{-}}$ free simulations (black lines with shadings indicating one standard deviation) averaged over measurement period at each site. The simulation, METsite_LAImerra, is driven with meteorological forcings from MERRA and site measurements. The LAI is from MERRA. The name and location (longitude, latitude) are shown in the title. The land types include evergreen needleleaf forest (ENF), deciduous broadleaf forest (DBF), shrublands (SHR), grasslands (GRA), and croplands (CRO). 


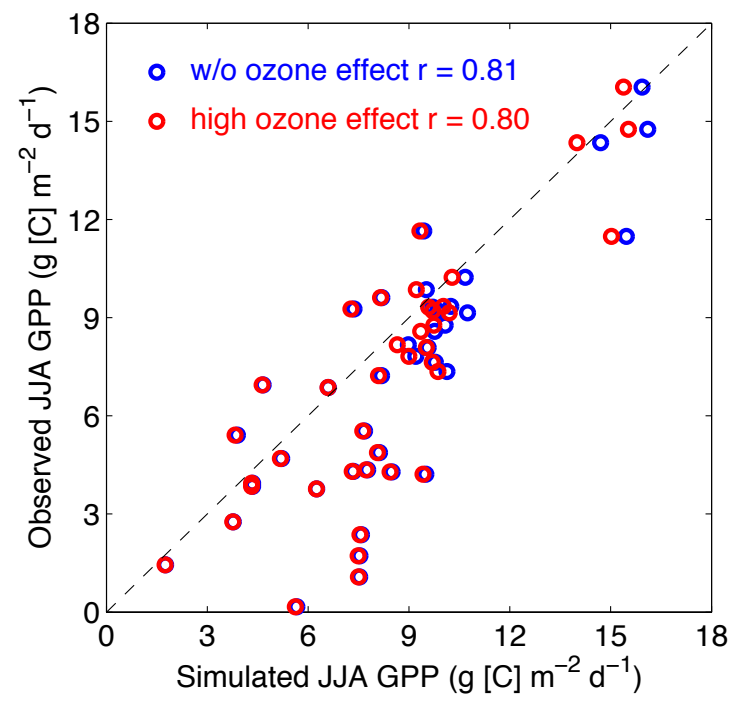

Fig. S5. Comparison of simulated summer average GPP without $\mathrm{O}_{3}$ damage (blue) and with $\mathrm{O}_{3}$ damage (red) with observations at 40 NACP sites. 
(a) $\chi^{2}$ for each site

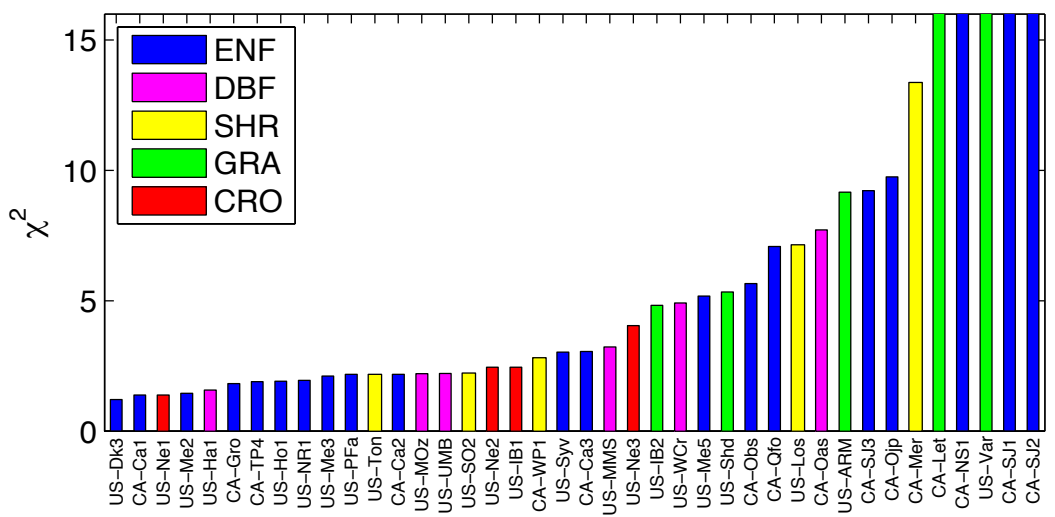

(b) $\Delta \chi^{2}$ for each site with low ozone effect

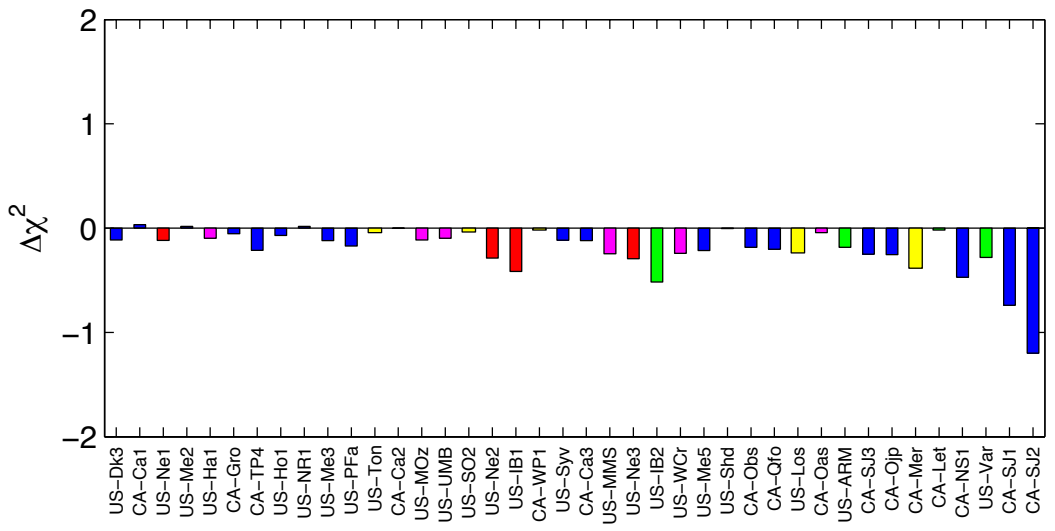

(c) $\Delta \chi^{2}$ for each site with high ozone effect

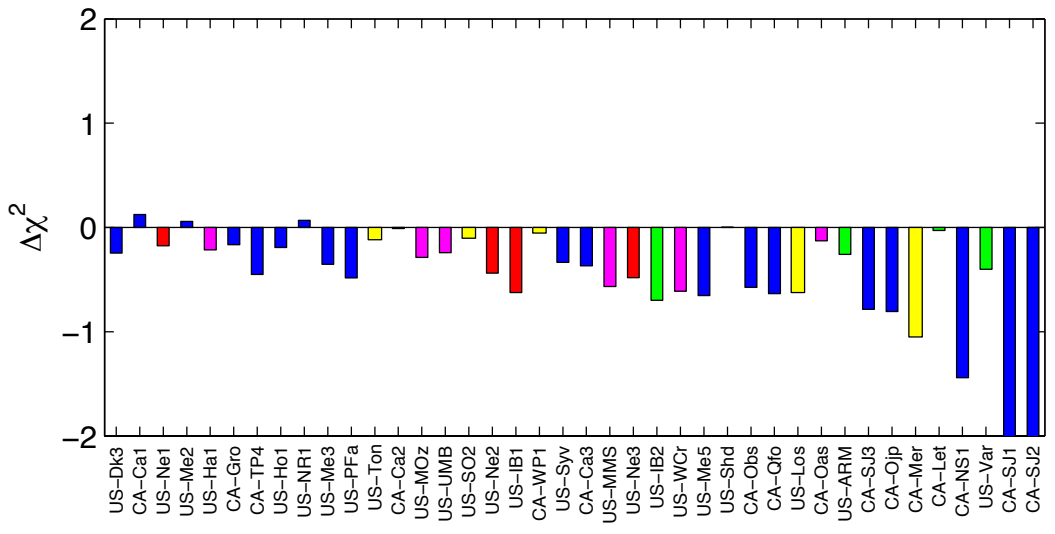

Fig. S6. The calculated (a) $\chi^{2}$ of GPP and changes in $\chi^{2}$ after the inclusion of $\mathrm{O}_{3}$ damage impact with (b) low and (c) high $\mathrm{O}_{3}$ sensitivity at each site. The sites are sorted according to the values of $\chi^{2}$ in (a). The land cover definitions are: GRA, Grasslands; CRO, Croplands; ENF, Evergreen Needleleaf Forest; DBF, Deciduous Broadleaf Forest; SHR, Shrubland. See section 2.2.1 for the definition of $\chi^{2}$. 
(a) JJA mean Ozone from GCM

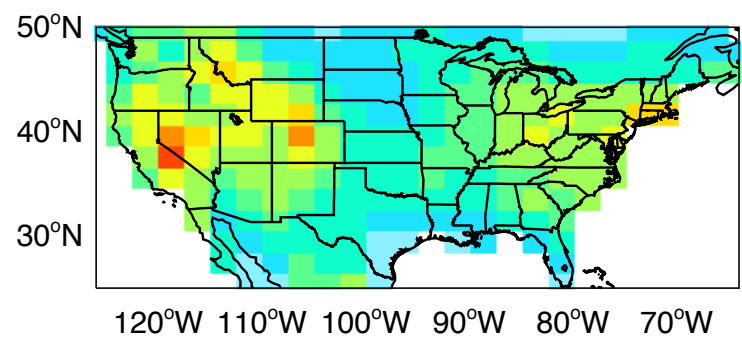

(c) JJA MDA8 Ozone from GCM

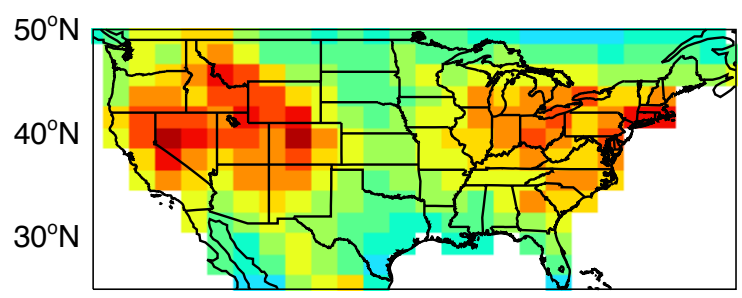

$120^{\circ} \mathrm{W} 110^{\circ} \mathrm{W} 100^{\circ} \mathrm{W} 90^{\circ} \mathrm{W} \quad 80^{\circ} \mathrm{W} \quad 70^{\circ} \mathrm{W}$ (b) JJA mean Ozone from CASTNET

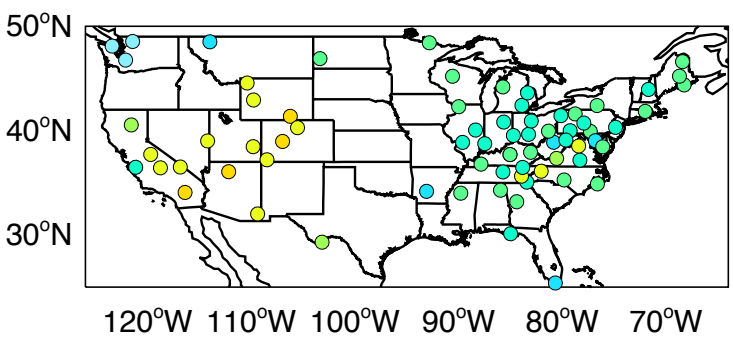

(d) JJA MDA8 Ozone from AIRDATA

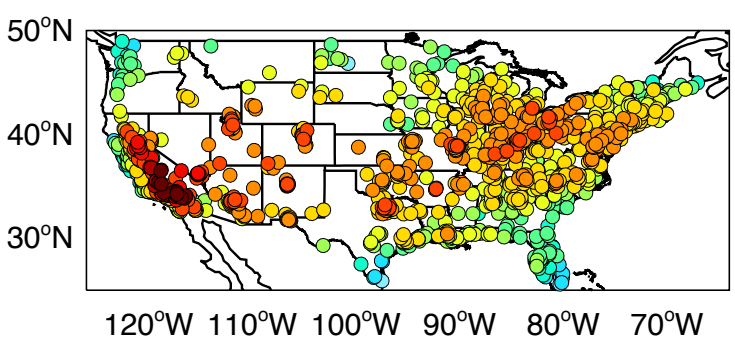

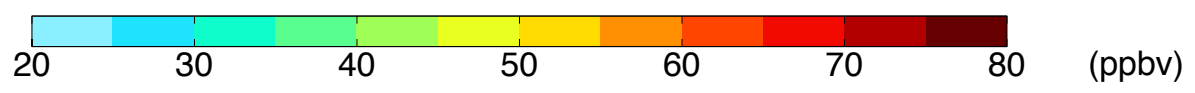

Fig. S7. Comparison of summertime surface (a, b) diurnal mean and (c, d) daily maximum 8-hour average ozone between (a, c) GCM and observations from (b) CASTNET and (d) AIRDATA. 
(a) JJA GPP with high ozone damage

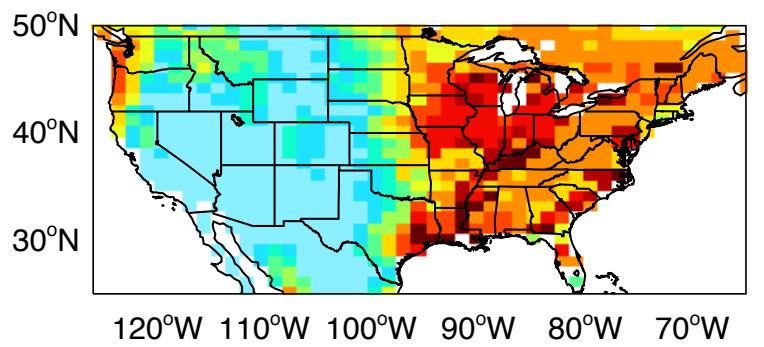

(b) JJA GPP from NACP sites

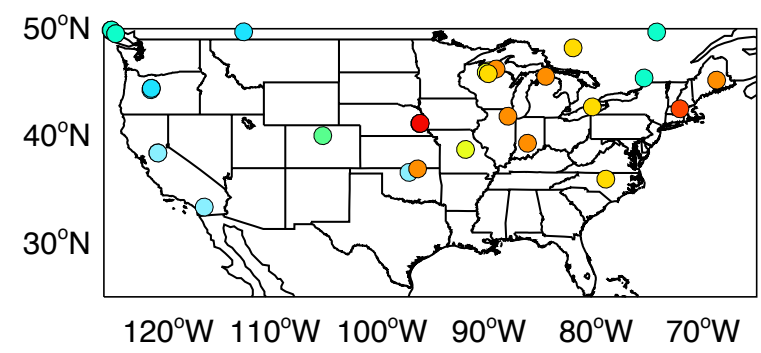

$\left(\mathrm{g} \mathrm{m}^{-2}\right.$ day $\left.^{-1}\right)$

Fig. S8. Comparison of summertime GPP from (a) simulations with high ozone damage effects and (b) in situ measurements from NACP. 